\title{
A TWO-SIDED H. LEWY EXTENSION PHENOMENON
}

\author{
L. R. HUNT ${ }^{1}$ AND M. KAZLOW ${ }^{2}$
}

\begin{abstract}
Let $M$ be a $C^{\infty}$ real $(n+k)$-dimensional CR-manifold in $\mathbf{C}^{n}$. We are interested in finding conditions on $M$ near a point $p \in M$ which imply that all CR-function on $M$ extend to holomorphic functions in some fixed neighborhood of $p$ in $\mathbf{C}^{n}$. Of course if $\boldsymbol{M}$ is a real hypersurface, it is known that $M$ having eigenvalues of opposite sign in its Levi form at $p$ will give us such an extension result. If we view the Levi form at a point on a general CR-manifold $M$ as a quadratic map from the holomorphic tangent space to the normal space of the real tangent space in $\mathbf{C}^{n}$, and if this map is surjective, then we prove our CR-functions extend to holomorphic functions in an open neighborhood of the point. We also show that if the real codimension of $M$ in $\mathbf{C}^{n}$ is 2 , and if the Levi form is totally indefinite, then the Levi form is onto $\mathbf{R}^{2}$ as a quadratic map, and hence we have our extension theory.
\end{abstract}

0. Introduction. Let $M$ be a connected locally closed $C^{\infty}$ submanifold of $\mathrm{C}^{n}$. A CR-function on $M$ is a $C^{\infty}$ solution of the tangential Cauchy-Riemann equations on $M$. Every holomorphic function on a neighborhood of $M$ when restricted to $M$ is a CR-function. The converse is false, as seen from the example of $M=\mathbf{R}^{n} \oplus i 0 \subset \mathbf{R}^{n} \oplus i \mathbf{R}^{n}=\mathbf{C}^{n}$. In this case, the tangential Cauchy-Riemann equations are trivial so that every $C^{\infty}$ function on $\mathbf{R}^{n} \oplus i 0$ is a CR-function.

There exists a complex analog of the second fundamental form for real submanifolds of $\mathbf{C}^{n}$ called the Levi form. When $M$ is a real hypersurface in $\mathbf{C}^{n}$, the Levi form is a real-valued sesquilinear form. If the Levi form at a point $p \in M$ has eigenvalues of opposite sign, then every CR-function on $M$ near $p$ can be extended to a holomorphic function on both sides of $M$ near $p$ [7, p. 51]. By elliptic regularity the CR-functions on $M$ near $p$ extend to holomorphic functions in a neighborhood of $p$ in $\mathbf{C}^{n}$.

If $M$ is a real submanifold of codimension 2 in $\mathbf{C}^{n}$, then the Levi form is a $\mathbf{R}^{2}$-valued sesquilinear form. If the orthogonal projection of the Levi form of $M$, at $p \in M$, on every line in $\mathbf{R}^{2}$ is a sesquilinear form with eigenvalues of opposite sign, we show every CR-function on $M$ near $p$ extends to a holomorphic function in a neighborhood of $p$.

We conclude this introduction with an outline of this paper. $\$ 1$ contains the

Presented to the Society, January 4, 1978; received by the editors November 3, 1977.

AMS (MOS) subject classifications (1970). Primary 32D10, $32 \mathrm{~F} 99$.

Key words and phrases. CR-functions, Levi form, quadratic maps, totally indefinite, CRextension.

${ }^{1}$ Partially supported by NSF Grant MCS 76-05267 at Texas Tech University.

2 Partially supported by NSF Grant MPS 75-05270 at Rice University.

(C) 1979 American Mathe matical Society 0002-9939/79/0000-0166/\$02.50 
definition of technical terms. In $\$ 2$ we prove that if the image of the Levi form at a point $p$ on a CR-manifold $M$ is the normal space, then the CR-functions on $M$ near $p$ extend to holomorphic functions near $p$. $\$ 3$ contains a proof that the image of an $\mathbf{R}^{2}$-valued quadratic form, whose orthogonal projection on any line is a quadratic form with eigenvalues of opposite sign, is $\mathbf{R}^{\mathbf{2}}$. Hence the $\mathbf{C R}$-functions on a codimension 2 submanifold of $\mathbf{C}^{n}$, whose Levi form has eigenvalues of opposite sign in every direction, extend to holomorphic functions since the image of the Levi form is the normal space. The authors would like to thank Mark Ferris for the present form of Lemma 3.3 and other discussion about quadratic maps.

1. Technical terms. If $M$ is a $C^{\infty}$ real $(n+k)$-dimensional submanifold of $\mathrm{C}^{n}$, by $T(M)$ we denote the real tangent bundle to $M$ with fiber $M_{p}$ for $p \in M$. By $H_{p}$ we denote the holomorphic tangent space at $p$, which is the largest complex subspace of $\mathbf{C}_{p}^{n}$ contained in $M_{p}$. If the dimension of $H_{p}$ is a constant function of $p \in M$, then $M$ is a $C R$-manifold with holomorphic tangent bundle $H(M)=\cup_{p \in M} H_{p}$. If $\operatorname{dim}_{\mathrm{C}} H_{p}$ equals $k$ then $M$ is a generic manifold.

If $M$ is a CR-manifold with $H_{p} \neq\{0\}$, then we define the Levi form at $p$, $L_{p}: H_{p} \mapsto \mathrm{C}_{p}^{n} / M_{p}$ by

$$
L_{p}(t)=\mathrm{II}_{p}(t, t)+\mathrm{II}_{p}\left(J_{p} t, J_{p} t\right)
$$

where $\mathrm{II}_{p}$ is the second fundamental form of $M$ at $p$ as a submanifold of $\mathbf{C}^{n}$ with the usual metric [4], [5] and $J_{p}$ is the almost complex structure tensor of $\mathbf{C}^{n}$ at $p$. We identify $\mathbf{C}_{p}^{n} / M_{p}$ with $M_{p}{ }^{\perp}$ and endow $M_{p}{ }^{\perp}$ with the induced inner product. If $\nu \in M_{p}^{\perp}$ then by $L_{p} \cdot \nu$ we denote the Levi form in the (normal) direction $\nu$. We set $L_{p} \cdot \nu(t)=L_{p}(t) \cdot \nu$, where $t \in H_{p}(M)$. A complex-valued function $f$ on $M$ is a $C R$-function if the differential of $f, f_{* p}: M_{p} \mapsto \mathbf{C}_{p}$ is complex linear when restricted to $H_{p}$ for all $p \in M$ [3].

2. An extension theorem. Our first result concerning CR-extension in every normal definition depends on the relation of the Levi form to $M_{p}{ }^{\perp}$.

Theorem 2.1. Let $M$ be a $C^{\infty}$ real $(n+k)$-dimensional $C R$-manifold in $\mathbf{C}^{n}$. If there exists a point $p$ in $M$ such that $L_{p}\left(H_{p}\right)=M_{p}{ }^{\perp}$, then there exists a neighborhood $U$ of $p$ in $M$ so that every $C R$-function on $U$ uniquely extends to a holomorphic function on a fixed neighborhood $\tilde{U}$ of $p$ in $\mathbf{C}^{n}$.

Proof. The hypothesis implies that $L_{p} \cdot \nu$ has eigenvalues of opposite sign for all $\nu \in M_{p}{ }^{\perp}$. The CR-manifold $M$ is generic, otherwise there exists $\nu \in M_{p}{ }^{\perp}$ such that $L_{p} \cdot \nu=0$ [9]. The hypothesis $L_{p}\left(H_{p}\right)=M_{p}{ }^{\perp}$ implies the existence of two $C^{\infty}$ Bishop analytic disc manifolds [1] (see also [3], [6], [8], and [11]) $M_{\nu}^{+}$and $M_{\nu}^{-}$for every $\nu \in M_{p}^{\perp}$. The analytic disc manifolds have the following properties (see [6]):

(1) $M_{\nu}^{ \pm}=M_{\nu}^{ \pm} \cup M$ is a real $(n+k+1)$-dimensional manifold with boundary such that $\pm \nu \in\left(M_{\nu}^{ \pm}\right)_{p}$. 
(2) $\nu$ points "inward" with respect to $M_{\nu}^{+}$and "outward" with respect to $M_{\nu}^{-}$.

The methods of R. Nirenberg [13] show that the CR-functions on $M$ near $p$ can be uniformly approximated by germs of holomorphic functions on compact subsets of $\boldsymbol{M}$. Furthermore, these germs of holomorphic functions extend to $M_{\nu}^{+}$and $M_{\nu}^{-}$and converge uniformly on compact subsets of $M_{\nu}^{+}$ and $M_{\nu}{ }^{-}$. The union of the $M_{\nu}{ }^{ \pm}$for every $\nu \in M_{p}{ }^{\perp}$ is dense in some neighborhood $\tilde{U}$ of $p$ in $\mathbf{C}^{n}$. This follows from the facts that $L_{p}\left(H_{p}\right)=M_{p}{ }^{\perp}$ and the analytic discs can be constructed in a way so that they depend smoothly on the normal directions at $p$. Therefore the germs of holomorphic functions that uniformly approximate a given $C R$-function on $M$ near $p$ actually converge uniformly on compact subsets of $\tilde{U}$ to a holomorphic function. Q.E.D.

REMARK. The above theorem can be generalized under the weaker assumption that $L_{p}\left(H_{p}\right)$ is dense in $M_{p}^{\perp}$.

3. Quadratic maps. Let $V$ be a real vector space and $(W, \cdot)$ a real inner product space. A quadratic map $Q: V \mapsto W$ is a function such that

$$
L^{Q}\left(v_{1}, v_{2}\right)=\frac{1}{4}\left[Q\left(v_{1}+v_{2}\right)-Q\left(v_{1}-v_{2}\right)\right] \text {, }
$$

with $v_{1}, v_{2} \in V$, is a symmetric bilinear map from $V$ to $W$, and such that $Q(\lambda v)=\lambda^{2} Q(v)$ for all $v \in V$ and $\lambda \in \mathbf{R}$. The map $Q \mapsto L^{Q}$ is a bijection between quadratic maps and symmetric bilinear maps. We say a quadratic map is totally indefinite if the quadratic function $v \rightarrow Q(v) \cdot w$ has eigenvalues of opposite sign [or equivalently $v \rightarrow Q(v) \cdot w$ is onto $\mathbf{R}$ ] for all nonzero $w \in W$. A quadratic map $Q$ is totally indefinite if and only if $Q(v)$ is not contained in any half-space.

We are interested in the properties of the ranges of certain $\mathbf{R}^{2}$-valued quadratic maps. These are given in the following three lemmas.

LemMa 3.1. Suppose $Q: \mathbf{R}^{2} \rightarrow \mathbf{R}^{2}$ is a quadratic map with the property that its image is not contained in a line in $\mathbf{R}^{2}$. Then the sets $Q\left(\mathbf{R}^{2}-\{0\}\right)$ and $Q\left(\mathbf{R}^{2}\right)-\{0\}$ are arcwise connected.

Proof. Since $\mathbf{R}^{2}-\{0\}$ is arcwise connected, it follows that $Q\left(\mathbf{R}^{2}-\{0\}\right)$ is arcwise connected. If $Q^{-1}(0)$ equals $\{0\}$, then $Q\left(\mathbf{R}^{2}-\{0\}\right)=Q\left(\mathbf{R}^{2}\right)-\{0\}$ is arcwise connected.

By an affine change of coordinates we may assume that $Q$ is equivalent to one of the three following quadratic maps:

(1) $Q\left(x_{1}, x_{2}\right)=\left(x_{1}^{2}, 2 b_{12} x_{1} x_{2}+b_{22} x_{2}^{2}\right)$,

(2) $Q\left(x_{1}, x_{2}\right)=\left(x_{1}^{2}+x_{2}^{2}, 2 b_{12} x_{1} x_{2}+b_{22} x_{2}^{2}\right)$,

(3) $Q\left(x_{1}, x_{2}\right)=\left(2 x_{1} x_{2}, b_{11} x_{1}^{2}+b_{22} x_{2}^{2}\right)$,

where $b_{11}, b_{12}$ and $b_{22}$ are real constants.

If $Q$ corresponds to (1) then either $Q^{-1}(0)=\{0\}$ or $b_{22}=0$. If $b_{22}=0$ then it is easy to check that $Q\left(\mathbf{R}^{2}\right)=\left\{\left(x_{1}, x_{2}\right) \mid x_{1} \supsetneqq 0\right\} \cup\{0\}$ so that $Q\left(\mathbf{R}^{2}\right)$ 
- $\{0\}$ is arcwise connected. If $Q$ corresponds to (2) then $Q^{-1}(0)=\{0\}$. If $Q$ corresponds to (3) and $b_{11} b_{22} \neq 0$ then $Q^{-1}(0)=\{0\}$. If $b_{11}$ and $b_{22}$ both equal zero, then $Q(v)$ is one dimensional, and if only one of $b_{11}$ and $b_{22}$ equals zero, then $Q$ is equivalent to a quadratic map of type (1). Q.E.D.

LEMMA 3.2. If $Q: \mathbf{R}^{2} \rightarrow \mathbf{R}^{2}$ is a totally indefinite quadratic map, then $Q\left(\mathbf{R}^{2}\right)=\mathbf{R}^{2}$.

PRoof. If $Q$ is totally indefinite, then by an affine change of coordinates we may assume that

$$
Q\left(x_{1}, x_{2}\right)=\left(2 x_{1} x_{2}, b_{11} x_{1}^{2}+b_{22} x_{2}^{2}\right)
$$

with $b_{11}>0$ and $b_{22}<0$. As in Lemma 3.1 we have $Q^{-1}(0)=\{0\}$. Let $(a, b)$ be an arbitrary point in $\mathbf{R}^{2}$. If $a=0$ and $b \neq 0$, in order to have $Q\left(x_{1}, x_{2}\right)=$ $(a, b)$, we find that $x_{2}=0$ and $x_{1}=\left(b / b_{11}\right)^{1 / 2}$ or $x_{1}=0$ and $x_{2}=\left(b / b_{22}\right)^{1 / 2}$, depending on whether $b>0$ or $b<0$. If $a \neq 0$, then we must have that $x_{1} \neq 0$ and $x_{2} \neq 0$. The condition that $Q$ be onto $\mathbf{R}^{2}$ is satisfied by the fact that the point $\left(x_{1}, x_{2}\right)=(a / 2 c, c)$ with

$$
c=\left(\frac{b-\left(b^{2}-b_{22} b_{11} a^{2}\right)^{1 / 2}}{2 b_{22}}\right)^{1 / 2}
$$

maps to the point $(a, b)$ under $Q$. Q.E.D.

Remark. Suppose $Q: \mathbf{R}^{2} \rightarrow \mathbf{R}^{2}$ satisfies the hypothesis of Lemma 3.1. By that lemma, $Q\left(\mathbf{R}^{2}\right)-\{0\}$ is arcwise connected, and $Q\left(\mathbf{R}^{2}\right)$ is the cone generated by $Q\left(S^{1}\right)$, where $S^{1}$ is the unit sphere in $\mathbf{R}^{2}$. Therefore, either $Q\left(\mathbf{R}^{2}\right)=$ $\mathbf{R}^{2}$ or there exist two extremal rays emanating from the origin that bound $Q\left(\mathbf{R}^{2}\right)$. Of course Lemma 3.2 shows that such extremal rays cannot exist if $Q$ is totally indefinite. These facts are useful in the proof of our last lemma.

LeMmA 3.3. If $Q: \mathbf{R}^{n} \rightarrow \mathbf{R}^{2}$, where $n \geqslant 2$, is a totally indefinite quadratic map, then $Q\left(\mathbf{R}^{n}\right)=\mathbf{R}^{2}$.

Proof. From the definition of a totally indefinite quadratic map and by an affine coordinate change on $\mathbf{R}^{2}$ we may assume that $(1,0)$ and $(0,1)$ are elements of $Q\left(\mathbf{R}^{n}\right)$ and that there exists a point $p$ in the third quadrant in $\mathbf{R}^{2}$ with $p \in Q\left(\mathbf{R}^{2}\right)$.

If there exist two points $\alpha$ and $\beta$ in $\mathbf{R}^{2}$ such that:

(i) $Q(\alpha)=(1,0)$ and $Q(\beta)=(0,1)$ or

(ii) $Q(\alpha)=(1,0)$ and $Q(\beta)=p$ or

(iii) $Q(\alpha)=(0,1)$ and $Q(\beta)=p$ and

$Q$ restricted to the plane in $\mathbf{R}^{n}$ determined by $\alpha$ and $\beta$ is totally indefinite, then $Q$ is onto $\mathbf{R}^{2}$ by Lemma 3.2. If such points do not exist, then by the remark preceding the lemma $Q\left(\mathbf{R}^{n}\right)$ contains the points in the smaller sectors in $\mathbf{R}^{2}$ determined by the rays (from the origin) through $(1,0)$ and $(0,1),(0,1)$ 
and $p$ and $(1,0)$ and $p$. Since the union of these sectors is $\mathbf{R}^{2}$ we have $Q\left(\mathbf{R}^{n}\right)=\mathbf{R}^{2}$. Q.E.D.

REMARK. It can be shown that the image of a quadratic map from $\mathbf{R}^{2}$ to $\mathbf{R}^{2}$ can be

(a) any ray emanating from the origin;

(b) any line through the origin;

(c) any open half-space union with the origin;

(d) $\mathbf{R}^{2}$;

(e) any closed sector $S$ such that $S-\{0\}$ is contained in a half-space.

We note that the Levi form at a point on a $C^{\infty}$ real $(n+k)$-dimensional CR-submanifold of real codimension 2 in $\mathbf{C}^{n}$ is a quadratic map with range contained in $\mathbf{R}^{2}$. Thus Theorem 2.1 and Lemma 3.3 imply the main result of this paper.

THEOREM 3.4. Let $M$ be a codimension 2 CR-submanifold in $\mathbf{C}^{n}$ such that the Levi form at $p \in M$ is totally indefinite. Then there exists a neighborhood $U$ of $p$ in $M$ so that every $C R$-function on $U$ uniquely extends to a holomorphic function on a fixed neighborhood $\tilde{U}$ of $p$ in $\mathbf{C}^{n}$.

In [12], Naruki proves estimates which show that all CR-distributions on a CR-submanifold $M$ of $\mathbf{C}^{n}$ near a point $p \in M$ at which $L_{p}$ is totally indefinite are $C^{\infty}$ CR-functions on $M$ near $p$. Thus Theorems 2.1 and 3.4 are also true for CR-distributions. For more details see [10].

Results in the direction of Theorem 3.4 have been proved by D. Ellis and C. D. Hill, and related theorems are mentioned in [2].

We give an example of a $C^{\infty}$ real 10 -dimensional CR-submanifold $M$ in $\mathbf{C}^{6}$ which satisfies the hypothesis of Theorem 3.4 at the origin (in $M$ and $\mathbf{C}^{6}$ ). Consider $M$ given by the equations $\left(z_{1}, \ldots, z_{6}\right.$ are the coordinates for $\mathbf{C}^{6}$, and $x_{1}, x_{2}, u_{1}, v_{1}, \ldots, u_{4}, v_{4}$ are the coordinates for $M$ ).

$$
\begin{aligned}
& z_{1}=x_{1}+i\left(z_{3} \bar{z}_{3}-z_{4} \bar{z}_{4}\right), \quad z_{2}=x_{2}+i\left(z_{5} \bar{z}_{5}-z_{6} \bar{z}_{6}\right), \\
& z_{3}=u_{1}+i v_{1}, \quad z_{4}=u_{2}+i v_{2}, \quad z_{5}=u_{3}+i v_{3}, \quad z_{6}=u_{4}+i v_{4} .
\end{aligned}
$$

As $u_{1}, v_{1}, u_{2}, v_{2}, u_{3}, v_{3}$, and $u_{4}, v_{4}$ vary over $\mathbf{R}^{8}$, then the map

$$
\left(u_{1}, v_{1}, u_{2}, v_{2}, u_{3}, v_{3}, u_{4}, v_{4}\right) \rightarrow\left(z_{3} \bar{z}_{3}-z_{4} \bar{z}_{4}, z_{5} \bar{z}_{5}-z_{6} \bar{z}_{6}\right)
$$

is onto $\mathbf{R}^{2}$, and the Levi form is totally indefinite.

Of course it is easy to construct an example of a CR-submanifold which does not satisfy the hypothesis of our main result. Let $M$ be a CR-submanifold of $\mathbf{C}^{n}$ which is given as the transversal intersection of two $C^{\infty}$ real hypersurfaces in $\mathbf{C}^{n}$, at least one of which is strictly pseudoconvex at $p \in M$.

\section{REFERENCES}

1. E. Bishop, Differentiable manifolds in complex Euclidean space, Duke Math. J. 32 (1965), $1-22$.

2. D. Ellis, C. D. Hill and C. C. Seabury, The maximum modulus principle. I. Necessary conditions, Indiana J. Math. 25 (1976), 709-717. 
3. S. J. Greenfield, Cauchy-Riemann equations in several variables, Ann. Scuola Norm. Sup. Pisa 22 (1968), 275-314.

4. R. Hermann, Convexity and pseudoconvexity for complex manifolds, J. Math. Mech. 43 (1964), 667-672.

5. Convexity and pseudoconvexity for complex manifolds, J. Math. Mech. 43 (1964), 1065-1070.

6. C. D. Hill and G. Taiani, Families of analytic discs in $\mathbf{C}^{n}$ with boundaries on a prescribed CR-submanifold, Ann. Scuola Norm. Sup. Pisa 5 (1978), 327-380.

7. L. Hörmander, An introduction to complex analysis in several variables, 2 nd ed. NorthHolland, Amsterdam; American Elsevier, New York, 1973.

8. L. R. Hunt and R. O. Wells, Jr., Extensions of CR-functions, Amer. J. Math. 98 (1976), $805-820$.

9. __ Holomorphic extension for nongeneric CR-submanifolds, Proc. Sympos. Pure Math., vol. 27, part 2, Amer. Math. Soc., Providence, R.I., 1975, pp. 81-88.

10. L. R. Hunt and M. Kazlow, $A$ two-regular $H$. Lewy extension phenomenon (to appear).

11. M. Kazlow, CR-functions on tube manifolds, Trans. Amer. Math. Soc. (to appear).

12. I. Naruki, An analytic study of a pseudo-complex structure, Proc. Internat. Conf. Functional Anal. and Related Topics, (Tokyo 1969), Univ. of Tokyo Press, Tokyo, pp. 72-82.

13. R. Nirenberg, On the H. Lewy extension phenomenon, Trans. Amer. Math. Soc. 168 (1972), 337-356.

Department of Mathematics, Texas Tech University, Lubbock, Texas 79409 (Current address of $\mathbf{L}$. R. Hunt)

Department of Mathematics, Rice University, Houston, Texas 77001

Current address (M. Kazlow): Department of Mathematics, Rutgers University, New Brunswick, New Jersey 08903 